\title{
Effect of Ground Plane Deformation on Electrical Performance of Air Microstrip Antennas
}

\author{
Wei Wang ${ }^{D}$, Yanjun Wang ${ }^{D}$, Shunxi Lou, Shuo Zhang, and Yatian Zhou \\ Key Laboratory of Electronic Equipment Structure Design, Ministry of Education, Xidian University, Xi'an 710071, China \\ Correspondence should be addressed to Yanjun Wang; m13119192707_1@163.com
}

Received 6 August 2019; Revised 24 December 2019; Accepted 31 January 2020; Published 28 March 2020

Academic Editor: Giuseppina Monti

Copyright (C) 2020 Wei Wang et al. This is an open access article distributed under the Creative Commons Attribution License, which permits unrestricted use, distribution, and reproduction in any medium, provided the original work is properly cited.

\begin{abstract}
This paper covers the variation of electrical performance of air microstrip antennas due to its ground plane deformation. A typical sinusoidal function is utilized to characterize the shape of deformation, based on which different shapes of ground plane can be obtained by changing control parameters. From the simulation results by HFSS, there is a strong linear relationship between the offset of resonant frequency and the amplitude of deformation. The equivalent thickness of the air medium is introduced into the calculation model, and then the mathematical model is established to describe the resonant frequency offset and deformation amplitude. It can also be seen from the radiation pattern that the directivity decreases with the increase of deformation. The proposed method can be used to predict the effect of ground plane deformation on electrical performance of air microstrip antennas.
\end{abstract}

\section{Introduction}

Microstrip antennas have many advantages, such as small size, light weight, low profile, conformal to carrier, etc. They have been widely used in communication, radar, and other fields [1]. However, at the same time they have disadvantages such as low efficiency, narrow bandwidth, and low gain if using normal substrate like FR-4 [2]. The thicker substrate and smaller dielectric constant are advantageous for antenna performance, which results in higher efficiency and gain [3-5]. Therefore, air medium can be added in practice, and the introduction of air layer reduces the equivalent dielectric constant and $Q$ value of microstrip antennas, thus achieving the purpose of increasing bandwidth. In the manufacturing process of microstrip antennas, small deviation of physical size often causes the change of effective electrical size, and the inhomogeneity of dielectric substrate, which will lead to the deviation of the antenna electrical performance from ideal situation. For example, for microstrip antennas working in Beidou band [6-8], because of the narrow bandwidth, there is a high demand for the accuracy of working frequency. Small change in the external environment may make resonant frequency offset, leading to the antenna not working properly. In addition, the structural deformation caused by external loads will also change the electrical performance of the antenna during its service. For example, microstrip antennas are widely used in spaceborne synthetic aperture radar (SAR) antenna $[9,10]$. The antenna in orbit is in a very complex thermal environment [11]. The thermal distortion caused by temperature change is the main reason for the change of microstrip antennas flatness. Another example is that the antenna working in the moving platform will be affected by air dynamic load and carrier vibration. These external loads will cause the flatness of the antenna, and the electrical performance of the antenna is closely related to flatness [12-14]. The flatness error will cause resonant frequency offset, impedance mismatch, gain loss, and sidelobe level increase [15-17]. Therefore, it is necessary to study the effect of structure deformation on the electrical performance of microstrip antennas.

Many investigations have been done about the effect of structure deformation on electrical performance of phased array antennas [18-20]. The coupling model of structure and electromagnetic is established. However, the mathematical model does not consider the type of elements and ignores the change of the electrical performance of antenna elements 


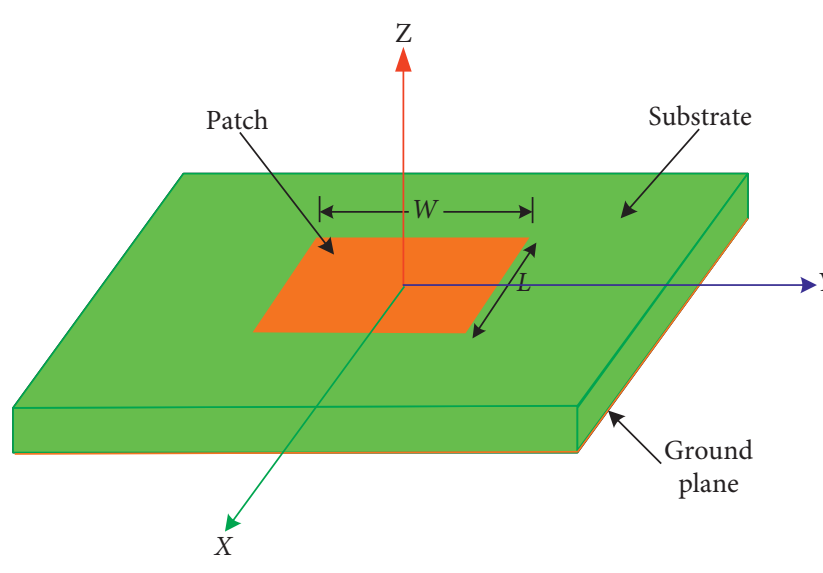

(a)

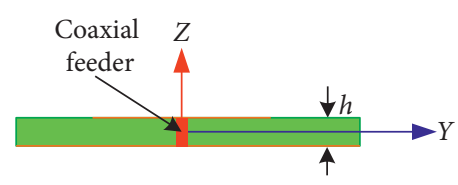

(b)

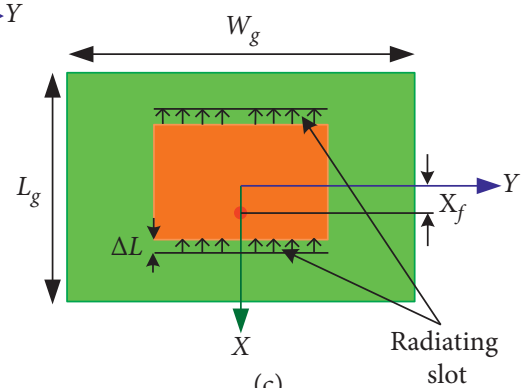

FIgURE 1: Geometric relationship of the microstrip antenna. The (a) three-dimensional diagram, (b) front view, and (c) top view.

after deformation. Moreover, only the position deviation between array elements is considered, and the pointing deviation of array elements and coupling are not considered. However, in practice, after the antenna structure is deformed, the radiation pattern of elements and coupling between elements will change. Others are studied from the perspective of conformal antenna, but only considering that microstrip antennas are cylindrical deformation, which is a very special type of deformation, so it is not universal [21-23]. In addition, the resonant frequency of patch antennas will be offset when it is stressed. Therefore, some related studies use patch antennas as sensors to detect structural strain [24-27].

The effect of array antenna deformation, conformal antenna, and stress-strain on the electrical performance is basically analyzed in the above research. However, the possibility of ground plane deformation is not considered. Therefore, it is necessary to study the effect of ground plane deformation on electrical performance of air microstrip antennas. First, HFSS electromagnetic simulation software is used to analyze the tendency of electrical performance of air microstrip antennas under different deformation states. Then, based on transmission-line model, the effect of physical parameters of rectangular patch antennas on resonant frequency is analyzed. At the same time, the effect of deformation on physical parameters of patch antennas is analyzed. Next, the influence mechanism between resonant frequency and deformation of air microstrip antennas is explored. The relationship between resonant frequency offset and ground plane deformation of air microstrip antennas is obtained. Finally, the effect of ground plane deformation on the radiation pattern is presented.

\section{Design Modeling and Deformation Definition}

Rectangular patch antenna is one of the most commonly used forms of microstrip antennas; the structure diagram is shown in Figure 1. The length of radiation patch is $L$, the width is $W$, and the thickness of dielectric substrate is $h$. Coaxial feed is one of the most common feeding methods for microstrip antennas.

The physical parameters of air microstrip antenna designed in this paper are shown in Table 1.

Through HFSS simulation, the electrical performance curves are shown in Figure 2.

When the ground plane deforms, as shown in Figure 3 , it can be seen that the patch and ground plane are no longer parallel. In this paper, a typical sinusoidal surface is selected to simulate the deformation of the ground plane, which forms a gradually changing state. Based on the sinusoidal surface given, the electrical performance of the antenna is analyzed when the ground plane deforms on the $X O Z$ plane and $Y O Z$ plane, respectively.

When the ground plane deforms on the $X O Z$ plane, the sinusoidal curve is

$$
z=A \sin (\omega x+\varphi)+t
$$

When the ground plane deforms on the $Y O Z$ plane, the sinusoidal curve is

$$
z=A \sin (\omega y+\varphi)+t
$$

From the sinusoidal curve, the shape of ground plane is affected by $A, \omega, \varphi$, and $t$. $A$ is amplitude of the sinusoidal curve and direct reflection of the ground plane deformation in vertical direction. $\omega$ is angular frequency, indirectly representing the number of deformed sinusoidal wave. $\varphi$ is phase, indirectly representing the left and right translation of the ground plane. And $t$ is up-down translation. $\omega$ can be defined by the following formula:

$$
\omega=\frac{2 \pi}{T} .
$$

When the ground plane deforms on the $X O Z$ plane, 
TABLE 1: Physical parameters of air microstrip antenna.

\begin{tabular}{lc}
\hline Parameters & Values $\left(\lambda_{0}\right.$ at $\left.2.45 \mathrm{GHz}\right)$ \\
\hline $\begin{array}{l}\text { Patch length, } L(\mathrm{~mm}) \\
\text { Patch width, } W(\mathrm{~mm})\end{array}$ & $55.6\left(0.454 \lambda_{0}\right)$ \\
$\begin{array}{l}\text { Ground plane length, } L_{g} \\
(\mathrm{~mm})\end{array}$ & $61.2\left(0.5 \lambda_{0}\right)$ \\
$\begin{array}{l}\text { Ground plane width, } \\
W_{g}(\mathrm{~mm})\end{array}$ & $100\left(0.82 \lambda_{0}\right)$ \\
$\begin{array}{l}\text { Air layer thickness, } h \\
(\mathrm{~mm})\end{array}$ & $5\left(0.041 \lambda_{0}\right)$ \\
$\begin{array}{l}\text { Dielectric constant, } \varepsilon_{r} \\
\text { Feeder position, } X_{f} \\
(\mathrm{~mm})\end{array}$ & 1.0006 \\
$\begin{array}{l}\text { Deformation amplitude, } \\
\text { (mm) }\end{array}$ & $14.2\left(0.008\left(0.020 \lambda_{0}\right), 1.5\left(0.012 \lambda_{0}\right), 3\left(0.0245 \lambda_{0}\right), 2.0 .016 \lambda_{0}\right)$, \\
& $\left.\lambda_{0}\right)$
\end{tabular}

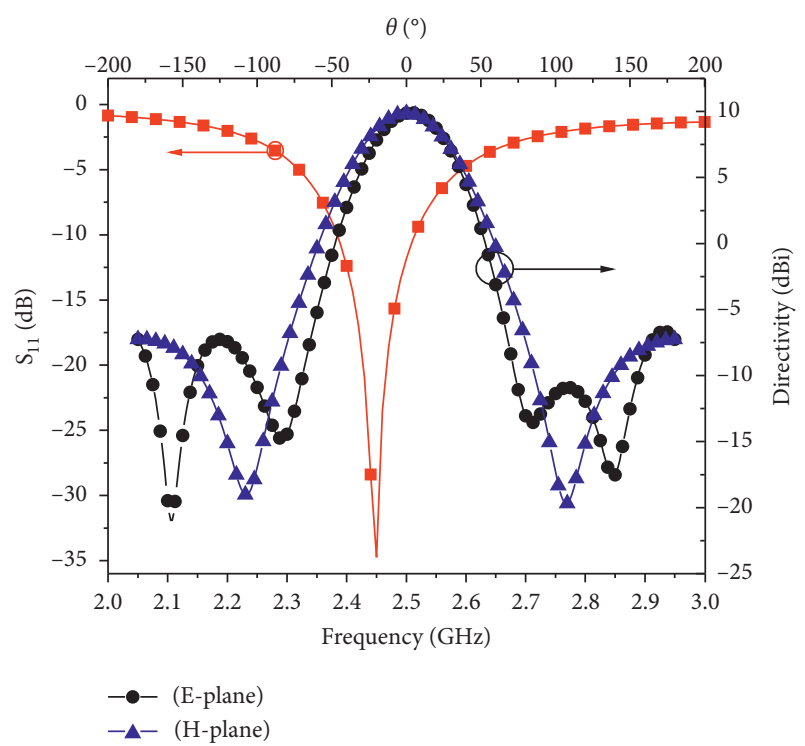

FIGURE 2: Simulation results of electrical performance of the air microstrip antenna.

$$
T=\frac{L_{g}}{N}
$$

When the ground plane deforms on the $Y O Z$ plane,

$$
T=\frac{W_{g}}{N}
$$

where $L_{g}$ and $W_{g}$ are length and width of the ground plane, respectively, which are about double size of the patch. Therefore, the period $T$ can be obtained according to sinusoidal wave numbers $N$ of the ground plane deformation, and then get $\omega$. The parameters of sinusoidal curve are shown in Table 2.

\section{Effect of Ground Plane Deformation on Resonant Frequency}

3.1. Resonant Frequency Offset and Equivalent Thickness. When the ground plane deforms on the XOZ plane, parameters of sinusoidal curve are shown in Table 2 . The relationship between resonant frequency and deformation amplitude $A$ under two $\omega$ values is analyzed, respectively, and the simulation results are shown in Figure 4.

As shown in Figure 4, when the ground plane deforms, the resonant frequency of the antenna shifts to right. In the vertical direction, with the increase of deformation amplitude $A$, the greater the resonant frequency offset, and the greater the return loss at center operating frequency. This indicates that the larger the deformation of the ground plane is, the antenna will be seriously mismatched, resulting in the antenna not working normally. In Figure 4(b), it can be seen that, with the ground plane deformation, the antenna return loss may be higher than $10 \mathrm{~dB}$.

Similarly, when the ground plane deforms on the YOZ plane, the simulation results are shown in Figure 5.

As shown in Figure 5(a), the number of the ground plane deformation sinusoidal wave is between half and one; the deviation of resonant frequency is small. In Figure 5(b), the sinusoidal wave number of the ground plane deformation is greater than one; the offset of resonant frequency is relatively large compared with the front case. Comparing Figure 4 with Figure 5, when the ground plane is deformed in different planes, the effect of $X O Z$ plane deformation on the resonant frequency is greater than that of $Y O Z$ plane; this could be explained by the fact that the $X O Z$ plane deformation affects the resonance path more significantly than the YOZ plane deformation does, particularly for the fundamental mode of resonance, as shown by the direction of the current plotted in Figures 6(a) and 6(b). Because the resonant frequency offset of microstrip antennas is mainly affected by the length and the width has little effect on resonant frequency, when the ground plane on the $X O Z$ deforms, it corresponds to the length direction of the patch; this may have a greater impact on resonant frequency. In addition, the position of coaxial feeder is not on the central line of the $X O Z$ plane, when the ground plane deforms on the $X O Z$ plane; this situation may also have a greater impact on resonant frequency.

From Figure 3, it can be seen that when the ground plane deforms, the thickness of air medium will be changed and it is an indeterminate value; therefore, the equivalent thickness $h^{\prime \prime}$ is introduced when calculating resonant frequency of the deformed antenna. $h^{\prime \prime}$ is defined as the thickness when the resonant frequency of the undeformed antenna is equal to the deformed antenna. The relationship between deformation amplitude $A$ and equivalent thickness $h^{\prime \prime}$ is shown in Figure 7.

3.2. Mathematical Model of Resonant Frequency Offset. In the actual manufacturing of microstrip antennas, due to the processing error of the antenna size and tolerance of substrate parameters, the electrical performance will change. If the error is small, the effect is mainly the change of resonant frequency. For patch antenna, its lowest resonant frequency [1] is

$$
f_{r}=\frac{c}{2 L^{\prime} \sqrt{\varepsilon_{e}}} \text {. }
$$

Due to fringing effects of microstrip line, the effective size of patch antenna is larger than physical size; length 


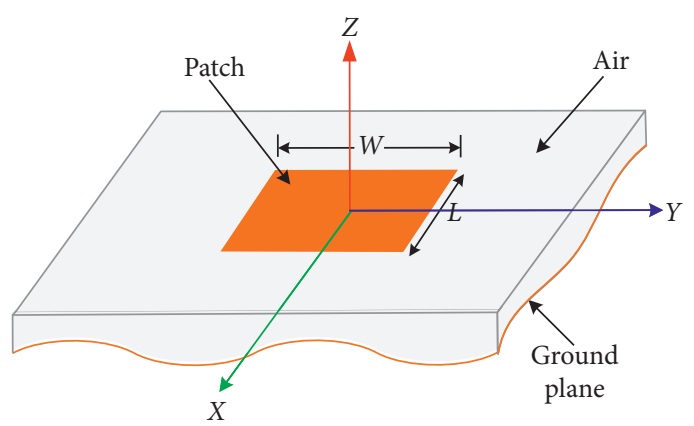

(a)

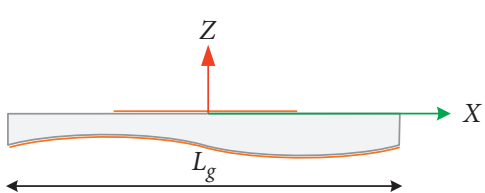

(b)

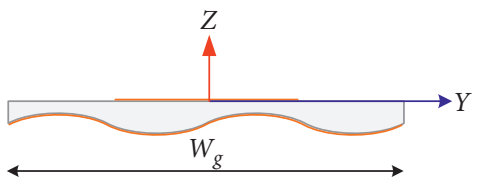

(c)

FIgURE 3: Ground plane deformation diagram. The (a) three-dimensional diagram, (b) XOZ plane deformation, and (c) YOZ plane deformation.

TABle 2: Parameters of sinusoidal curve with four different ground plane deformations.

\begin{tabular}{cccccc}
\hline \multicolumn{2}{l}{ Deformation cases } & Angular frequency, $\omega$ & Phase, $\varphi(\mathrm{rad})$ & Translation, $t(\mathrm{~mm})$ & Deformation amplitude, $A(\mathrm{~mm})$ \\
\hline \multirow{2}{*}{ XOZ } & Case a & $1 / 18$ & & 5 & $1-3.5$ \\
\multirow{2}{*}{ YOZ } & Case b & $1 / 9$ & $\pi / 2$ & 5 \\
& Case c & $1 / 30$ & & & \\
\hline
\end{tabular}

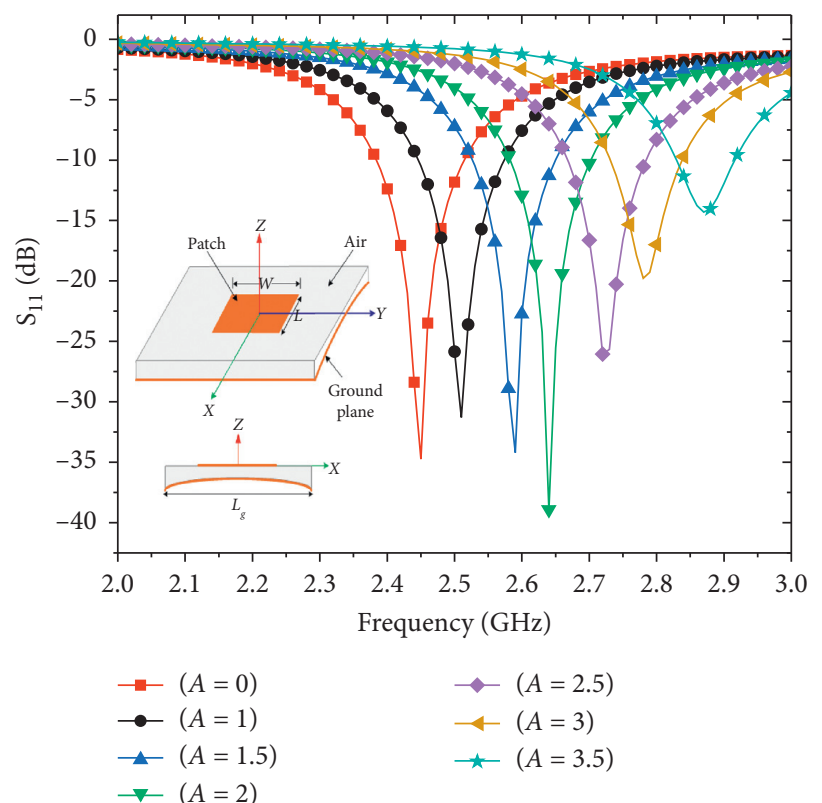

(a)

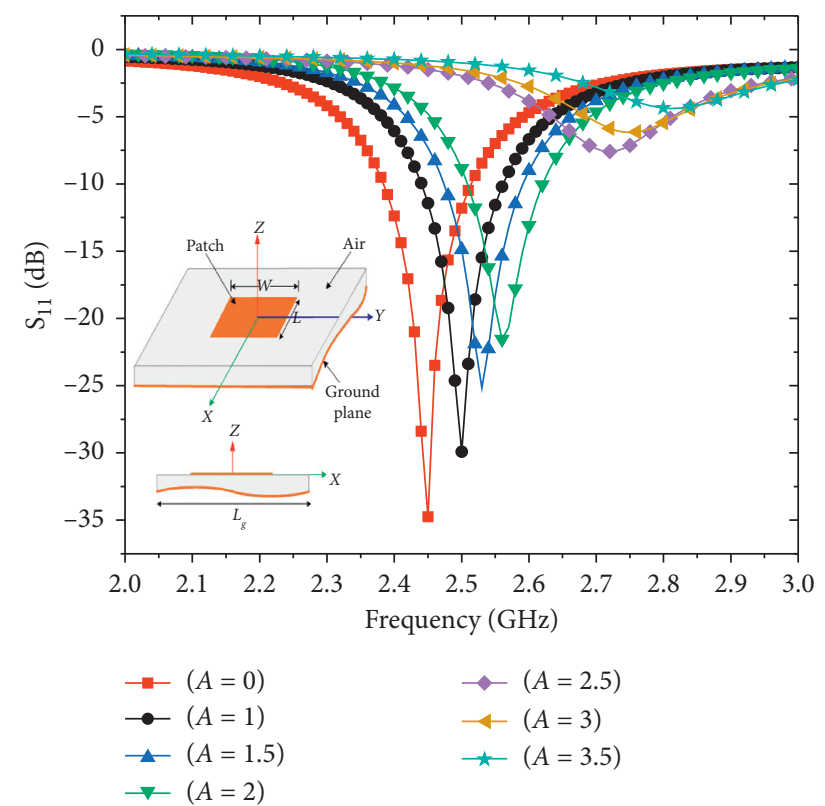

(b)

FIgURE 4: The variation of $S$ parameter with the deformation amplitude $A$ of $X O Z$ plane. (a) Case a and (b) case b.

compensation $\Delta L$ is required. The effective length $L^{\prime}$ of the patch can be expressed as the sum of the physical length $L$ and compensation length $\Delta L[1]$.

$$
\begin{aligned}
L^{\prime} & =L+2 \Delta L, \\
\Delta L & =0.412 h\left(\frac{\varepsilon_{e}+0.3}{\varepsilon_{e}-0.258}\right)\left(\frac{((W / h)+0.264)}{((W / h)+0.8)}\right),
\end{aligned}
$$

where $\varepsilon_{e}$ is the effective dielectric constant of the dielectric substrate and a simple empirical formula for the effective dielectric constant [1] is

$$
\varepsilon_{e}=\frac{\varepsilon_{r}+1}{2}+\frac{\varepsilon_{r}-1}{2}\left(1+12 \frac{h}{W}\right)^{1 / 2} .
$$

When the ground plane deforms, the thickness of air medium will be changed. And the thickness will affect the 


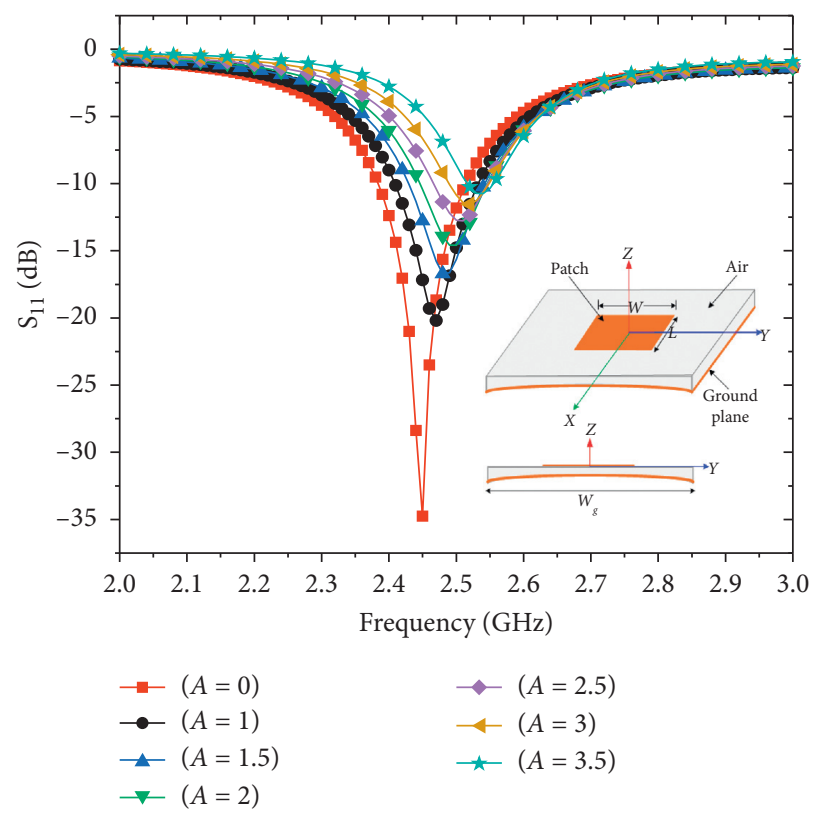

(a)

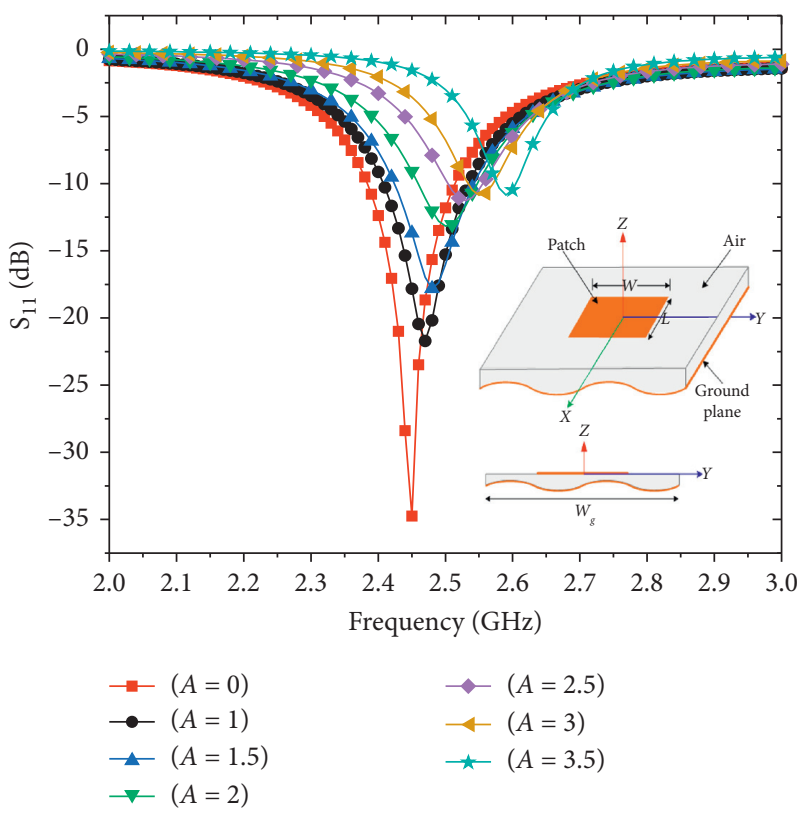

(b)

Figure 5: The variation of $S$ parameter with the deformation amplitude $A$ of $Y O Z$ plane. (a) Case $c$ and (b) case d.

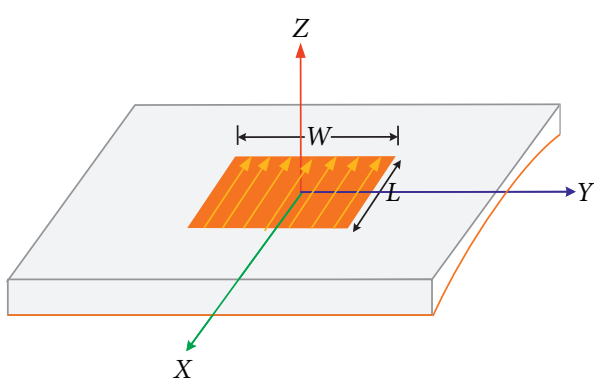

(a)

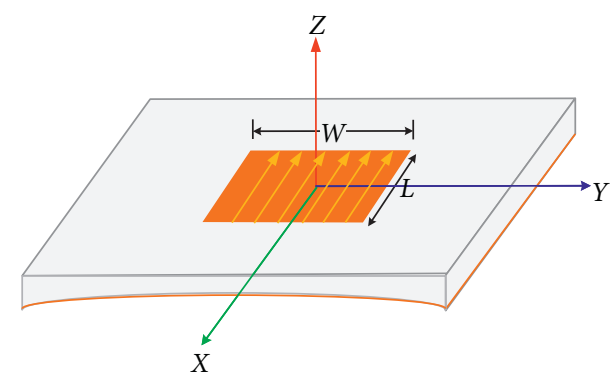

(b)

FIgURE 6: Current distribution of air microstrip antenna. (a) XOZ plane deformation. (b) YOZ plane deformation.

electrical size $L^{\prime}$ and $\varepsilon_{e}$, thus leading to the change of resonant frequency. Since $f_{r}$ is a function of $L^{\prime}$ and $\varepsilon_{e}$, therefore, the offset of resonant frequency can be derived from the partial derivatives of $f_{r}$ for $L^{\prime}$ and $\varepsilon_{e}$, respectively.

$$
\left\{\begin{array}{l}
\frac{\partial f_{r}}{\partial L^{\prime}}=\frac{c}{2 \sqrt{\varepsilon_{e}}}\left(-L^{\prime^{-2}}\right), \\
\frac{\partial f_{r}}{\partial \varepsilon_{e}}=\frac{c}{2 L^{\prime}}\left(-\frac{1}{2} \varepsilon_{e}^{-3 / 2}\right) .
\end{array}\right.
$$

According to the simultaneous (6) and (9), the relationship between resonant frequency offset and the change of physical parameters can be obtained as

$$
\left\{\begin{array}{l}
\frac{\partial f_{r}}{f_{r}}=-\frac{\partial L^{\prime}}{L^{\prime}} \\
\frac{\partial f_{r}}{f_{r}}=-\frac{1}{2} \frac{\partial \varepsilon_{e}}{\varepsilon_{e}}
\end{array}\right.
$$

Because $\varepsilon_{e}$ is an intermediate variable, it is related to $\varepsilon_{r}, h$, and $W$, so we can derive partial derivatives of $\varepsilon_{e}$ further.

$$
\left\{\begin{array}{l}
\frac{\partial \varepsilon_{e}}{\partial \varepsilon_{r}}=\frac{1}{2}\left[1+\left(1+12 \frac{h}{W}\right)^{-1 / 2}\right], \\
\frac{\partial \varepsilon_{e}}{\partial h}=-\frac{\varepsilon_{r}-1}{2} \frac{6}{W}\left(1+12 \frac{h}{W}\right)^{-3 / 2}, \\
\frac{\partial \varepsilon_{e}}{\partial W}=\frac{3 h}{W^{2}}\left(\varepsilon_{r}-1\right)\left(1+12 \frac{h}{W}\right)^{-3 / 2}
\end{array} .\right.
$$

According to the error statistics theory, if $f$ is the function of $x_{1}, x_{2}, \ldots, x_{N}, \Delta x_{1}, \Delta x_{2}, \ldots, \Delta x_{N}$ are independent standard error. Meanwhile, according to the variance synthesis theorem of independent variables, then the standard error of total result is 


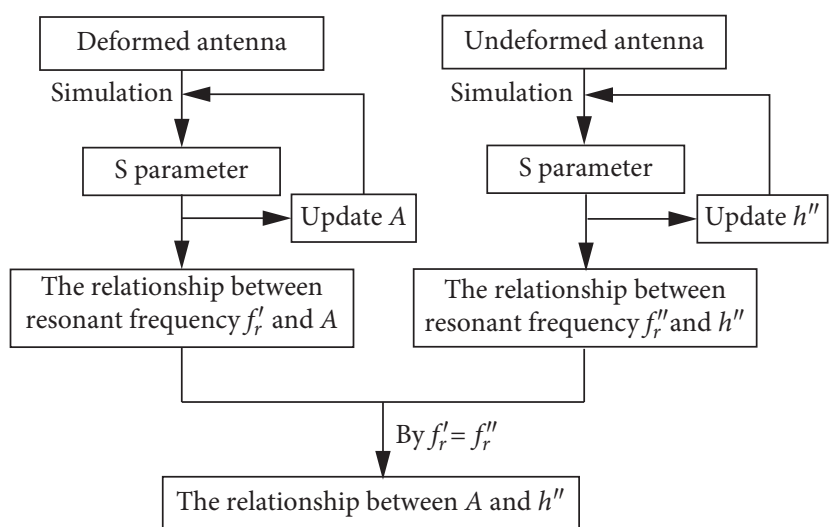

(a)

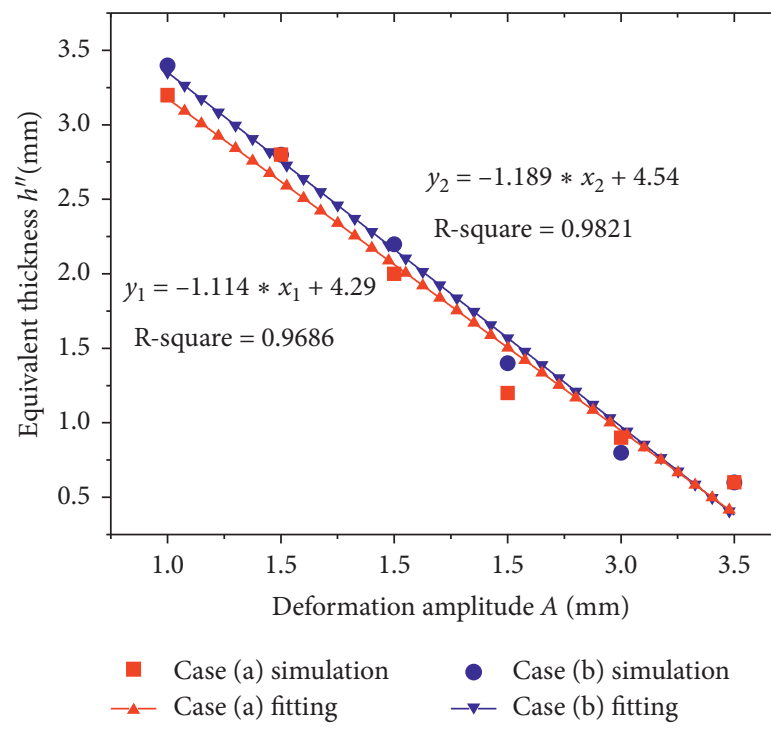

(b)

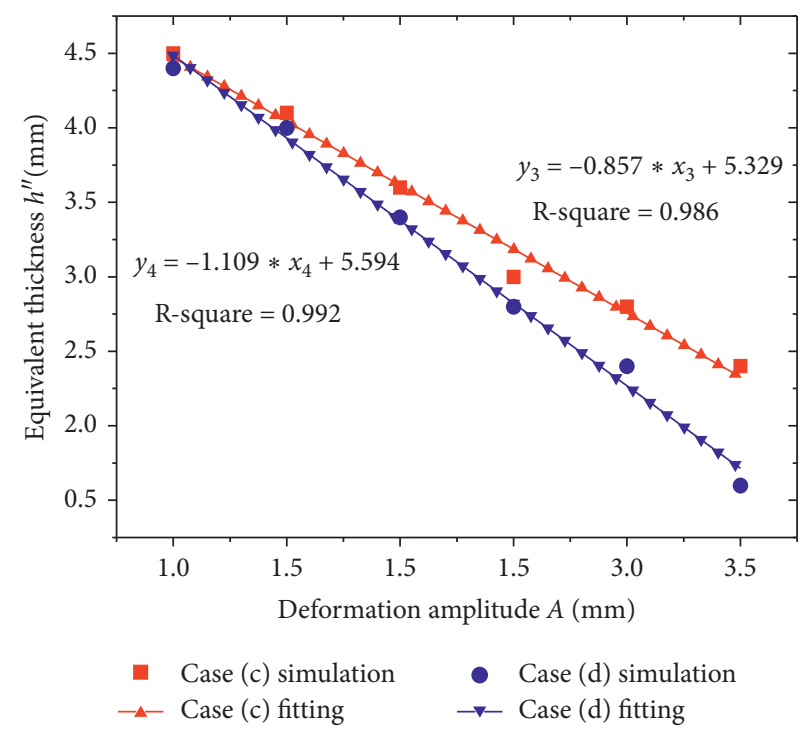

(c)

Figure 7: Relationship between deformation amplitude $A$ and equivalent thickness $h^{\prime \prime}$. The (a) equivalent thickness illustration, (b) XOZ plane deformation, and (c) YOZ plane deformation.

$$
\Delta f= \pm\left[\left(\frac{\partial f}{\partial x_{1}} \Delta x_{1}\right)^{2}+\left(\frac{\partial f}{\partial x_{2}} \Delta x_{2}\right)^{2}+\cdots+\left(\frac{\partial f}{\partial x_{N}} \Delta x_{N}\right)^{2}\right]^{1 / 2}
$$

Since $\varepsilon_{e}$ is a function of $\varepsilon_{r}, h$, and $W$, and the three variables are basic physical parameters and independent of each other, it can be obtained from (13)

$$
\left(\Delta \varepsilon_{e}\right)^{2}=\left(\frac{\partial \varepsilon_{e}}{\partial \varepsilon_{r}} \Delta \varepsilon_{r}\right)^{2}+\left(\frac{\partial \varepsilon_{e}}{\partial h} \Delta h\right)^{2}+\left(\frac{\partial \varepsilon_{e}}{\partial W} \Delta W\right)^{2}
$$

Thus, the relationship between the relative offset of resonant frequency and the change of physical parameters can be obtained as

$$
\frac{\left|\Delta f_{r}\right|}{f_{r}}=\left[w\left(\frac{\Delta L^{\prime}}{L^{\prime}}\right)^{2}+(1-w)\left(\frac{1}{2} \frac{\Delta \varepsilon_{e}}{\varepsilon_{e}}\right)^{2}\right]^{1 / 2}, \quad 0 \leq w \leq 1,
$$

$w$ is the weight coefficient. For air microstrip antenna, because the dielectric constant of air is about 1, the dielectric constant is basically unchanged by ground plane deformation, so $w=1$.

3.3. Numerical Results and Comparison. The resonant frequency can be solved by using previous mathematical model. The equivalent thickness $h^{\prime \prime}$ can be obtained from the deformation amplitude $A$ according to Figure 7 ; then the effective length $L^{\prime}$ of the patch can be obtained. The offset of resonant frequency $\Delta f_{r}$ can be obtained by applying the above mechanism model. The comparison between simulation and theoretical results is shown in Figure 8.

As can be seen from Figure 8, the theoretical results are generally in good agreement with simulation results, and there is a strong linear relationship when the number of the deformation sinusoidal wave is small. But when the deformation sinusoidal wave number is large, the shift of resonant frequency will change abruptly and the linearity is poor. 
3.4. Equivalent Circuit Model Interpretation. In this section, the bending effect of ground plane deformation is studied by using the generalized lumped element circuit model. The parameters of the lumped circuit with different deformation states are studied. The shift of resonant frequency is related to the change of the lumped circuit parameters. In addition, by combining the resonant circuit model linked with Optimization algorithm, the accurate $Z$ parameter over a wide frequency spectrum can be reconstructed with only a limited number of simulated frequency points required. Compared with the wideband sweep in the full wave simulation, the simulation time can be greatly reduced.

As shown in Figure 9, the circuit model topology has been used to study rectangular patch antennas. The orthogonal modes of resonance are modeled as the series connection of parallel resonant section. The high frequency inductance $L_{0}$ is used to simulate the impedance behavior of the antenna feed point in the upper and lower edge of the band of interest.

According to the topological structure of Figure 9, we get the general expression of the input impedance of the equivalent circuit

$$
Z_{\mathrm{eq}}=j 2 \pi f L_{0}+\sum_{i=1}^{m}\left(\frac{1}{R_{i}}+\frac{1}{j 2 \pi f L_{i}}+j 2 \pi f C_{i}\right)^{-1},
$$

where $m$ represents the resonance number of the solution frequency band and $R_{i}, L_{i}$, and $C_{i}$ are the $i_{\text {th }}$ equivalent lumped resistance, inductance, and capacitance. In this paper, we choose $m=1$ and ignore the influence of higher order modes, since we are more interested in the frequency range where the first resonant is dominated. The parameters of the equivalent circuit are determined by the Optimization algorithm. A normalized error function has been defined as the fitness

$$
\frac{1}{Z_{0}}\left(\frac{1}{N} \sum_{k=1}^{N}\left\{\operatorname{Re}\left[Z_{s}\left(f_{k}\right)-Z_{e q}\left(f_{k}\right)\right]^{2}+\operatorname{Im}\left[Z_{s}\left(f_{k}\right)-Z_{e q}\left(f_{k}\right)\right]^{2}\right\}\right)^{1 / 2}
$$

where $Z_{0}$ is the characteristic impedance of $50 \Omega, N$ is the number of frequency sampling, $f_{k}$ is the frequency for the $k_{\text {th }}$ sample, and $Z_{s}$ and $Z_{\text {eq }}$ are the simulated and equivalent input impedance of the patch antenna. By minimizing the fitness function, Optimization algorithm finds the best parameters for the equivalent circuit model which generates the impedance curve closest to the simulation results over the frequency of interest. Four cases have been studied for deformation in $X O Z$ plane with $A=0$ (the flat case), $1 \mathrm{~mm}$, $1.5 \mathrm{~mm}$, and $2 \mathrm{~mm}$. The input impedance from the optimized circuit parameters demonstrates very good agreement with the full wave simulations for the four cases with different deformation, in a frequency range that starts from $2 \mathrm{GHz}$ and includes the first resonant mode. As can be seen from Figure 10, there is only one self-excited resonance point in the whole frequency band. So, there is no change in the antenna mode, it still works in the base mode, and no higher order mode appears.
Table 3 shows the equivalent circuit parameters of air microstrip antenna deformation in the above four cases. As expected from the simulation and analytical model, the effect of ground plane deformation on the first resonant mode has been observed by the circuit parameters $R_{1}, C_{1}$, and $L_{1}$. Compared to the flat case with $A=0$, at $A>0$, the capacitance $C_{1}$ increases and the inductance $L_{1}$ decreases. The LC product decreases, which results in an increased resonant frequency.

\section{Effect of Ground Plane Deformation on the Radiation Pattern}

When the ground plane deforms, the relationship between E-plane radiation pattern and deformation amplitude $A$ under four $\omega$ values is analyzed, respectively. The simulation results are shown in Figure 11.

As can be seen from the radiation patterns in Figure 11, when the ground plane deforms, the directivity decreases with the increase of the deformation amplitude $A$. The beam pointing of the E-plane pattern will shift to right. But when the ground plane deforms on the $Y O Z$ plane, the directivity decreases slightly relative to the deformation on the $X O Z$ plane.

Efficiency is also one of the important parameters for antennas. In order to reduce the length of the article and without affecting the conclusion, we only give the data of case a, as follows. The other three cases are similar to the case a, so they are omitted. Table 4 shows the variation of antenna efficiency with deformation amplitude $A$.

As can be seen from Table 4 , the radiation efficiency is slightly reduced and close to 1 . Because both the patch and ground plane are ideal conductors for analysis, there is no conductor loss. And the medium is air, so there is no dielectric loss. In conclusion, the antenna is lossless; the radiation efficiency is theoretically approximately 1 . However, the total efficiency of the antenna decreases with the increase of deformation amplitude $A$ due to the increase of return loss at the operating frequency. The above results again show that the antenna deformation has a relatively small effect on the radiation performance but can cause serious antenna mismatch.

\section{Measurement and Comparison}

The air microstrip antenna prototype is made by connecting the copper ( $0.5 \mathrm{~mm}$ thick) to the ground plane $(0.5 \mathrm{~mm}$ thick) with a liquid glue, as shown in Figure 12. The patch is attached to the ground plane by foam, providing firm connections and flexibility in bending. The size of the ground plane is $100 \mathrm{~mm} \times 120 \mathrm{~mm}$. In general, the ground plane size of the patch antenna should be 2-3 times the patch size, so as to maintain a good front and rear ratio. $S_{11}$ is measured by performing six bending deformations corresponding to amplitude $A=1 \sim 3.5 \mathrm{~mm}$; the step value is $0.5 \mathrm{~mm}$. The resonant frequency of air microstrip antenna with the ground plane deformation was measured by network analyzer. The same $S_{11}$ measurement was repeated three times to represent the measurement error. The average value of the resonant 


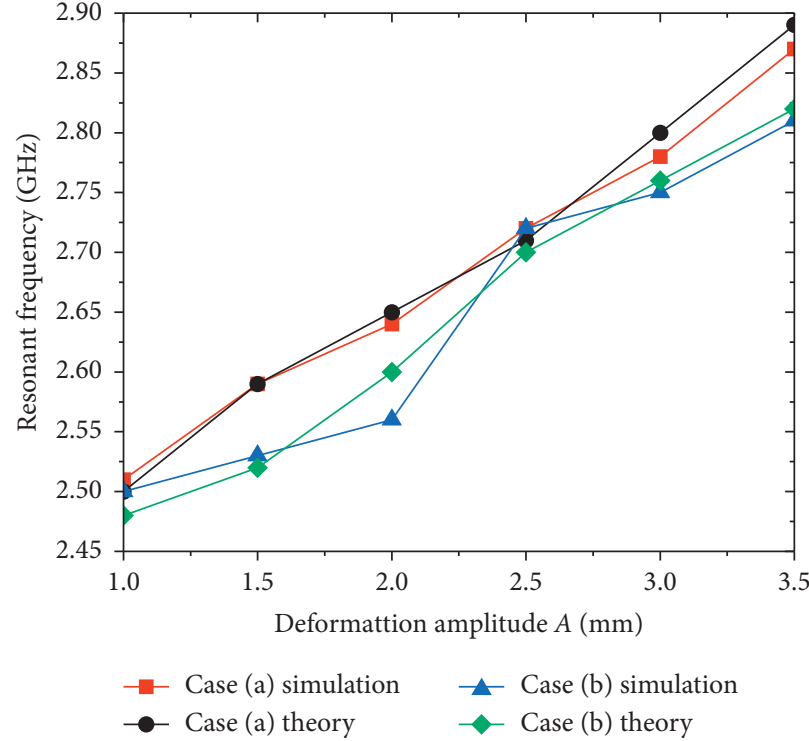

(a)

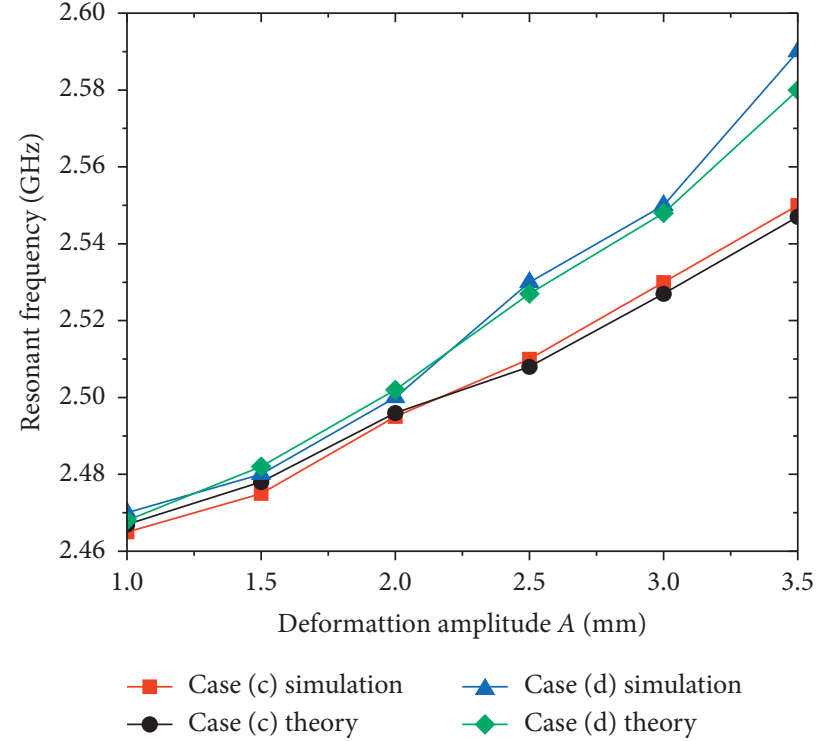

(b)

Figure 8: Comparison between simulation and theoretical results of resonant frequency. (a) XOZ plane deformation and (b) YOZ plane deformation.

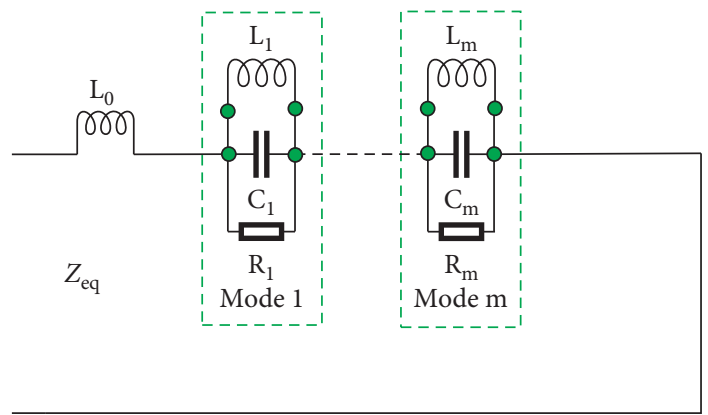

Figure 9: The equivalent circuit of the patch antenna.

frequency is measured. The measurement results show that it has good repeatability in three rounds measurement. As expected, The measurement results show that the resonant frequency shift of ground plane deformation in $X O Z$ plane is larger, while the influence of deformation in $Y O Z$ plane on resonant frequency is smaller, as shown in Figure 13. It is observed that when the bending deformation is large, the structure will change obviously, resulting in impedance maladjustment and bandwidth reduction. For ground plane deformation in $Y O Z$, the stability of input matching bandwidth is relatively small. As can be seen from Figure 14, the measured results are in good agreement with the simulation results. And it also proves the correctness of the conclusion.

\section{Conclusion}

This paper covers the electrical performance variation of air microstrip antenna under the ground plane deformation. When the ground plane deforms, the resonant frequency of the antenna will shift. When the ground plane deforms on two different planes, respectively, the offset of resonant

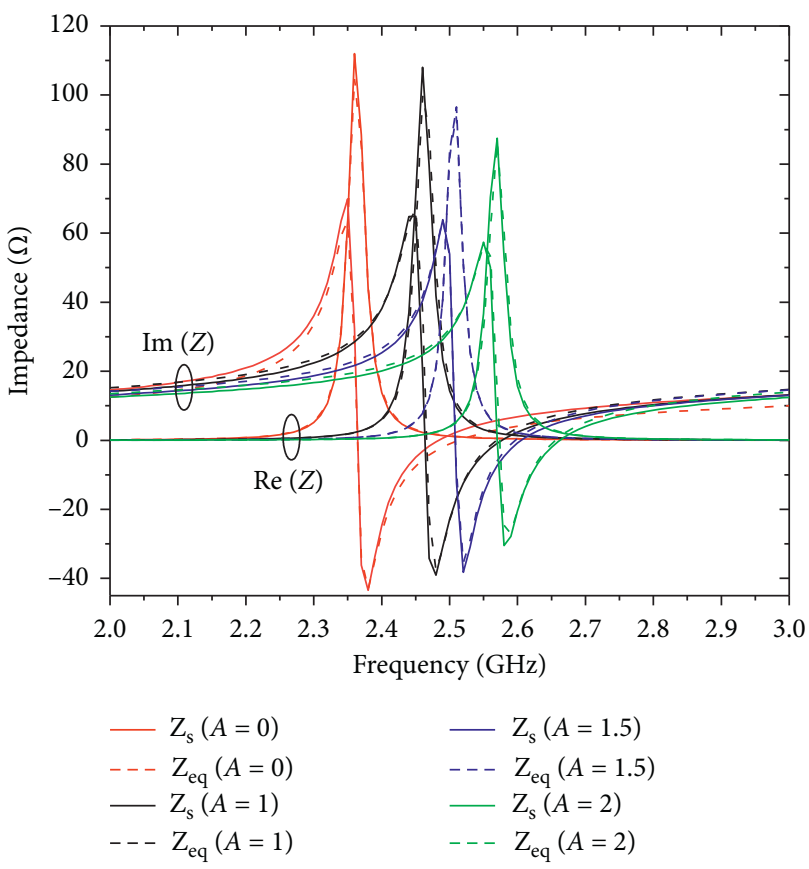

FIGURE 10: Input impedance from the optimized circuit parameters compared with the simulation results for the four cases with $A=0$ (the flat case), $1 \mathrm{~mm}, 1.5 \mathrm{~mm}$, and $2 \mathrm{~mm}$.

frequency on the $X O Z$ plane deformation is greater than that on the $Y O Z$ plane deformation. It also can be seen from the radiation pattern that the directivity of the antenna will decrease. When the ground plane deforms on $Y O Z$ plane, the loss of directivity is relatively small.

In a word, the deformation of ground plane of air microstrip antennas has great impact on electrical performance, which is not conducive to realize the electrical 
TABLE 3: Equivalent circuit model parameters for air microstrip antenna deformation in XOZ plane with $A=0$ (the flat case), $1 \mathrm{~mm}, 1.5 \mathrm{~mm}$, and $2 \mathrm{~mm}$.

\begin{tabular}{lcccc}
\hline \multirow{2}{*}{ Circuit parameters } & \multicolumn{3}{c}{ Deformation amplitude $A(\mathrm{~mm})$ (part of case a) } \\
& 0 & 1 & 1.5 & 2 \\
\hline$L_{0}(\mathrm{nH})$ & 0.843 & 0.875 & 0.852 & 103.5 \\
$R_{1}(\Omega)$ & 115.35 & 108.04 & 57.60 & 89.62 \\
$C_{1}(\mathrm{pF})$ & 50.532 & 53.635 & 0.070 & 60.03 \\
$L_{1}(\mathrm{nH})$ & 0.090 & 0.078 & 0.064 \\
\hline
\end{tabular}

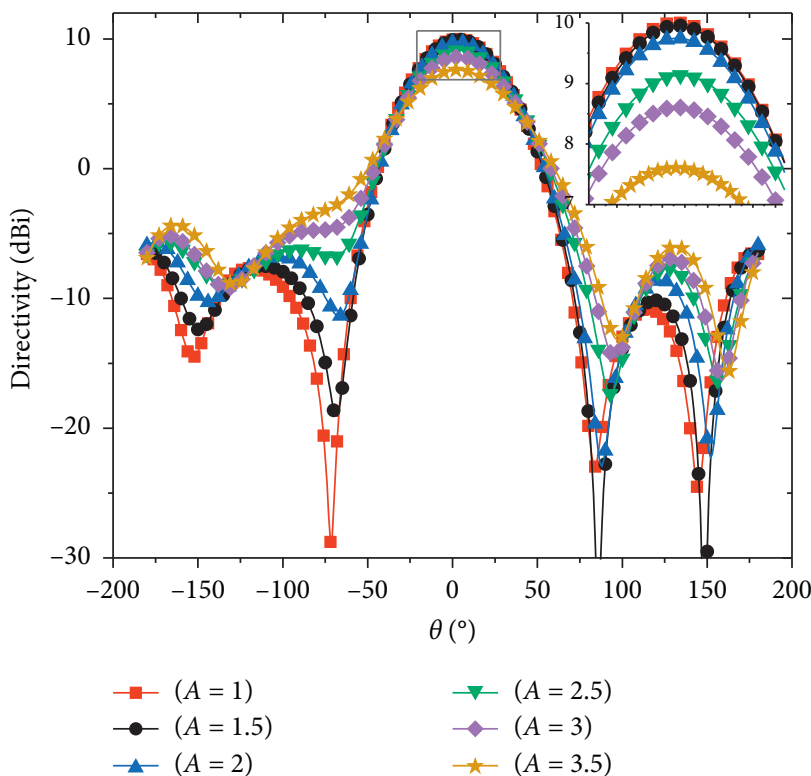

(a)

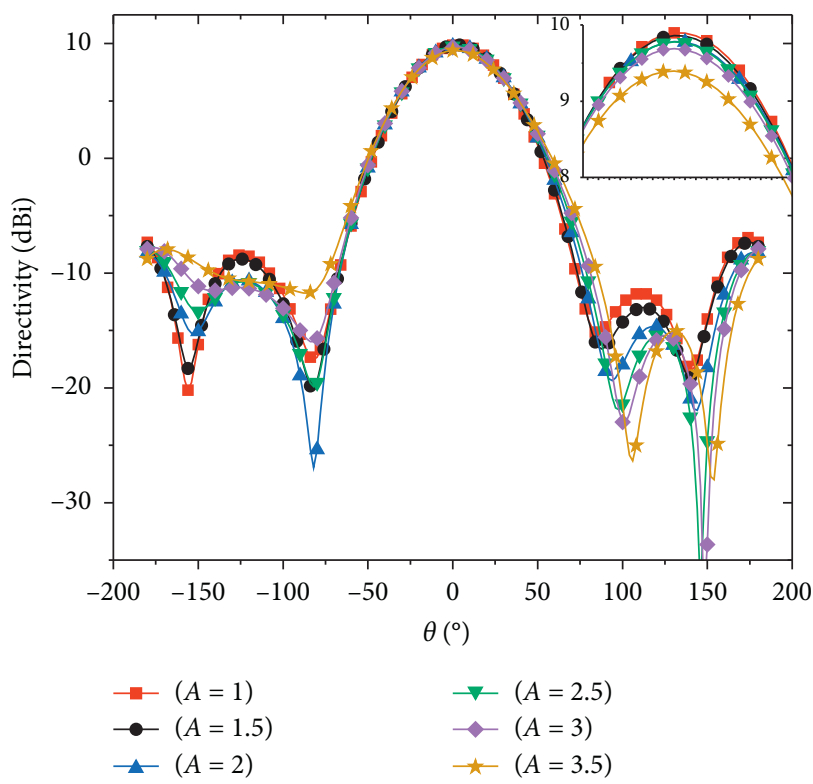

(c)

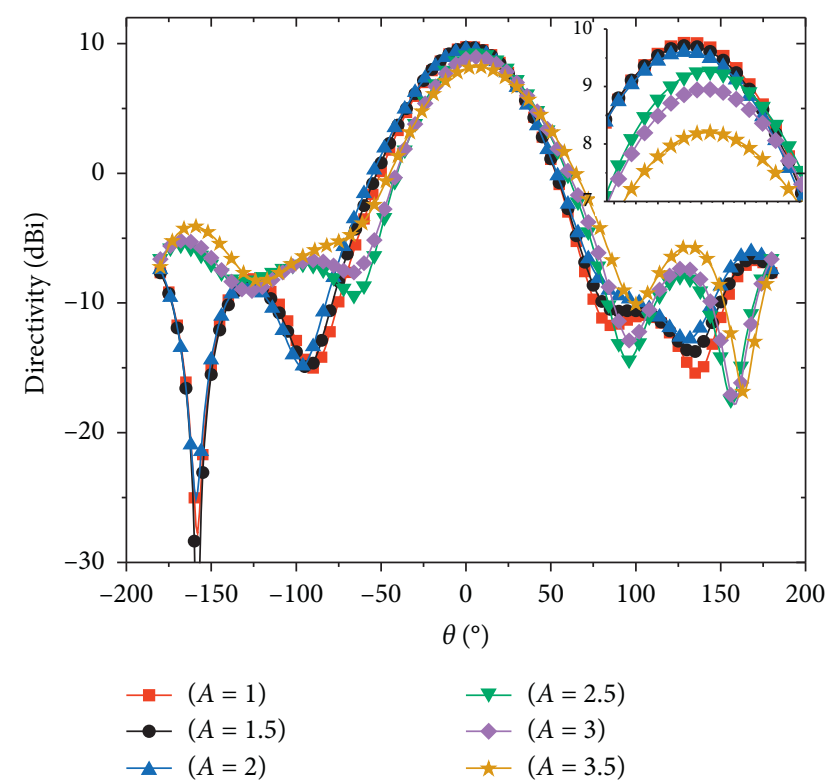

(b)

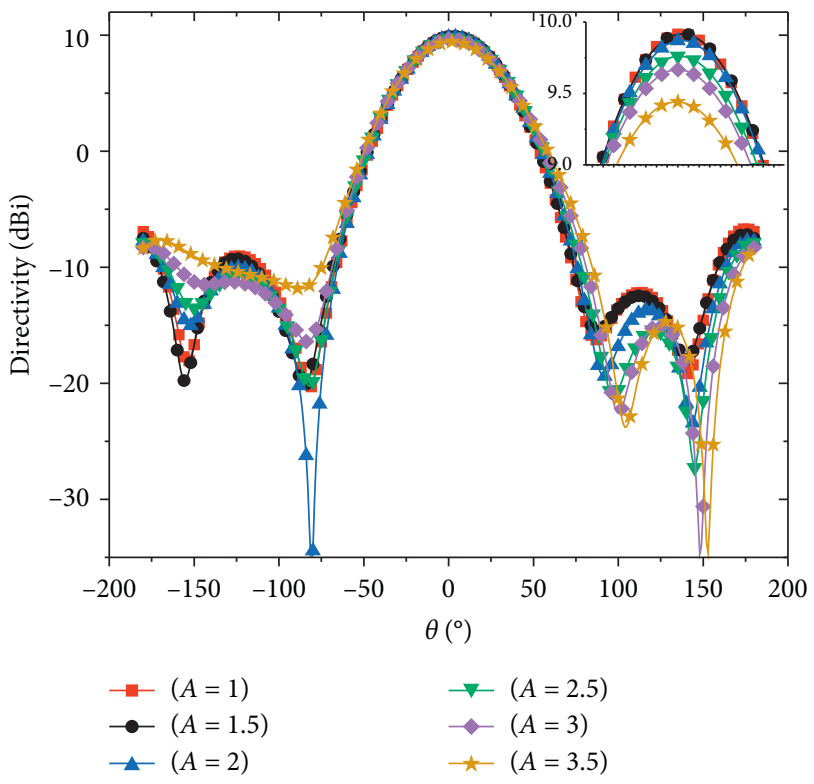

(d)

FIGURE 11: The E-plane radiation patterns change with deformation amplitude $A$. (a) Case a, (b) case b, (c) case c, and (d) case d.

performance. Therefore, in engineering practice, antenna structure deformation should be minimized to ensure that the antenna can work effectively. It can also be seen from the results that there is a good linear relationship between resonant frequency offset and deformation of air microstrip antennas. The effect of ground plane deformation on resonant frequency of air microstrip antennas can be effectively calculated by the mathematical model. 
TABLE 4: Efficiency of air microstrip antenna.

\begin{tabular}{|c|c|c|c|c|c|c|c|c|}
\hline $\begin{array}{l}\text { Deformation } \\
\text { cases }\end{array}$ & & $\begin{array}{c}\text { Angular } \\
\text { frequency } \omega\end{array}$ & $\begin{array}{c}\text { Deformation } \\
\text { amplitude } A(\mathrm{~mm})\end{array}$ & $\begin{array}{c}\text { Incident } \\
\text { power }(\mathrm{W})\end{array}$ & $\begin{array}{c}\text { Accepted } \\
\text { power }(\mathrm{mW})\end{array}$ & $\begin{array}{c}\text { Radiated } \\
\text { power }(\mathrm{mW})\end{array}$ & $\begin{array}{l}\text { Radiation } \\
\text { efficiency \% }\end{array}$ & $\begin{array}{c}\text { Total } \\
\text { efficiency \% }\end{array}$ \\
\hline \multirow{6}{*}{$X O Z$} & \multirow{6}{*}{$\begin{array}{c}\text { Case } \\
\text { a }\end{array}$} & \multirow{6}{*}{$1 / 18$} & 1 & 1 & 929.63 & 910.55 & 97.95 & 91.06 \\
\hline & & & 1.5 & 1 & 647.20 & 630.92 & 97.48 & 63.10 \\
\hline & & & 2 & 1 & 470.47 & 456.60 & 97.05 & 45.66 \\
\hline & & & 2.5 & 1 & 300.07 & 291.91 & 97.28 & 29.19 \\
\hline & & & 3 & 1 & 219.23 & 210.95 & 96.22 & 21.10 \\
\hline & & & 3.5 & 1 & 148.06 & 142.09 & 95.97 & 14.21 \\
\hline
\end{tabular}

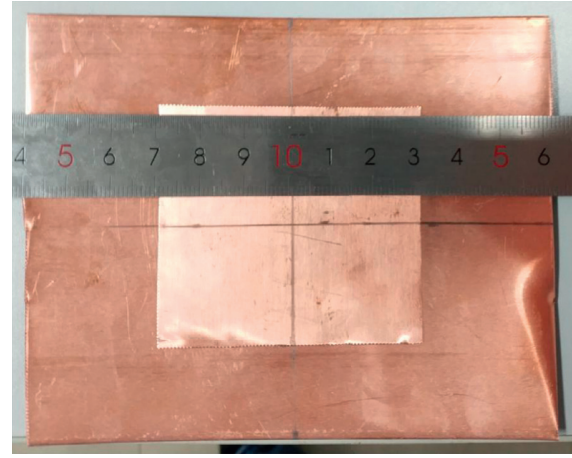

(a)

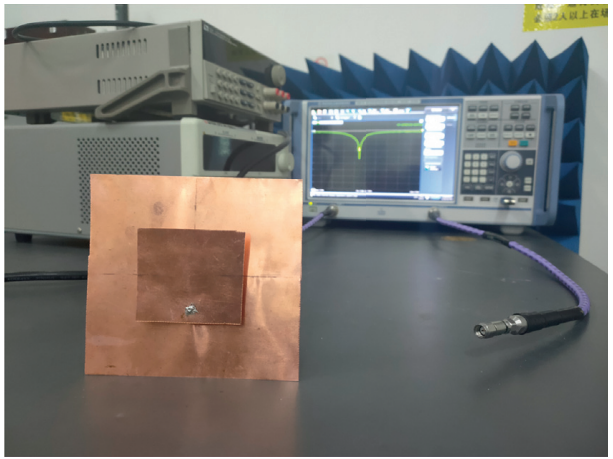

(b)

FIGURE 12: Fabricated air microstrip antenna. The (a) top view and (b) photo of the measured antenna prototype.

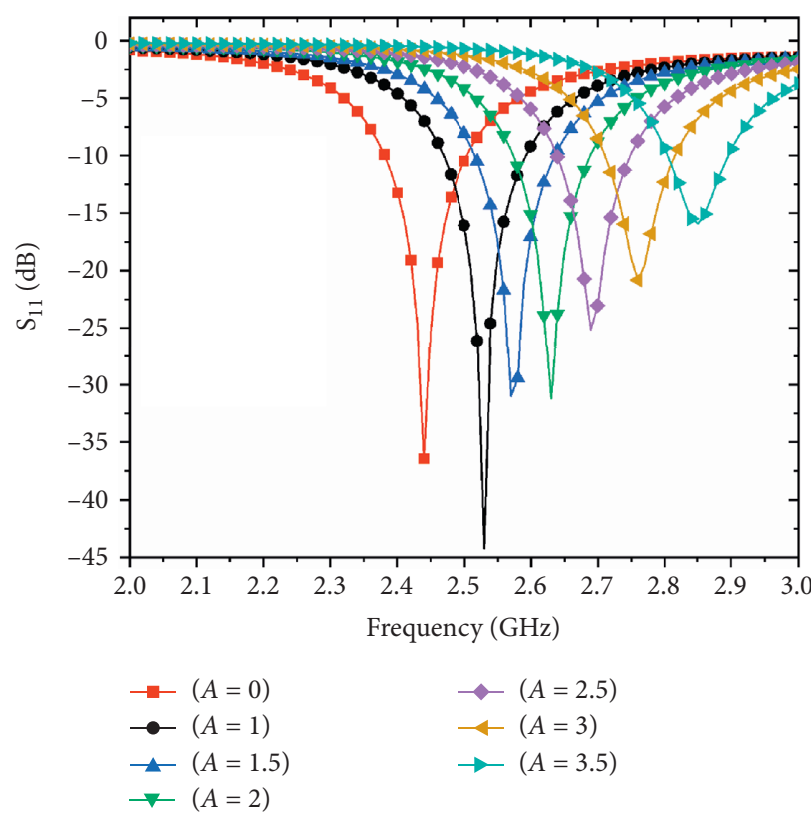

(a)

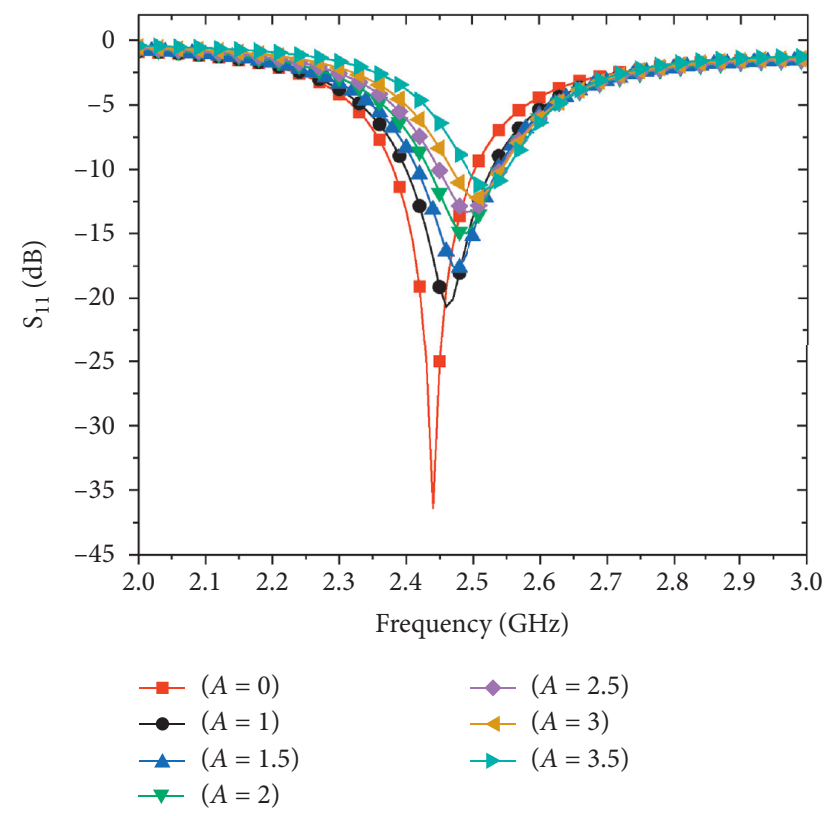

(b)

Figure 13: Measured results of $S$ parameter. (a) Case a and (b) case c. 


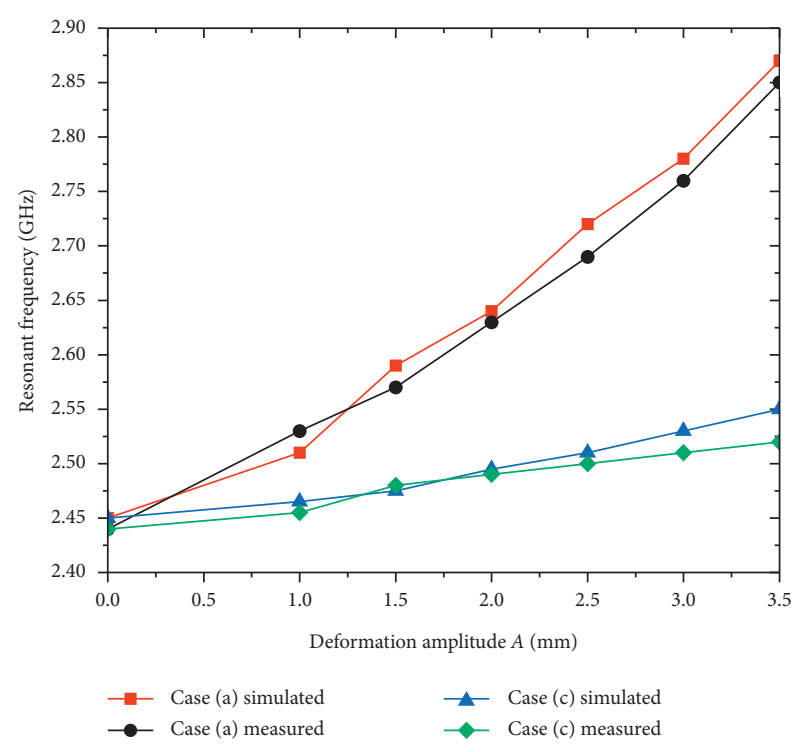

FIGURE 14: The simulation results are compared with the measured results.

In this paper, the effect of the ground plane deformation on electrical performance of air microstrip antennas is approximately processed. In practice, the antenna should be analyzed mechanically in ANSYS firstly, and then the deformed model is reconstructed in HFSS. Finally, the electrical performance of the deformed antenna is analyzed in HFSS. Therefore, in the next work, the mechanical analysis of air microstrip antenna in ANSYS will be consistent with the actual situation, including the study of more general medium microstrip antennas deformation on electrical performance of the antenna.

\section{Data Availability}

The data used to support the findings of this study are available from the corresponding author upon request.

\section{Conflicts of Interest}

The authors declare that they have no conflicts of interest.

\section{Acknowledgments}

This work was supported in part by the National Natural Science Foundation of China under Grant 51490661. The authors would like to thank Dr. Chaoliu Ge and Dr. Sihao Qian for their helpful insights.

\section{Supplementary Materials}

The supplementary material is an animation of the current distribution and electric field distribution of the air microstrip antenna with the deformation amplitude when the ground plane is deformed. (Supplementary Materials)

\section{References}

[1] C. A. Balanis, Antenna Theory: Analysis and Design, Wiley, Hoboken, NJ, USA, 3rd edition, 2005.

[2] M. T. Ali, H. Jaafar, S. Subahir, and A. L. Yusof, "Gain enhancement of air substrates at $5.8 \mathrm{GHz}$ for microstrip antenna array," in Proceedings of the 2012 Asia-Pacific Symposium on Electromagnetic Compatibility (APEMC), pp. 477-480, Singapore, May 2012.

[3] W.-W. Li, Q.-H. Li, Y. Meng, J.-Y. Wang, and W.-M. Xu, “A broadband microstrip patch antenna with multiple open slots," Microwave and Optical Technology Letters, vol. 61, no. 3, pp. 626-632, 2019.

[4] Y.-T. Liu, C.-W. Su, K.-L. Wong, and H.-T. Chen, "An airsubstrate narrow-patch microstrip antenna with high radiation performance for $2.4 \mathrm{GHz}$ WLAN access point," Microwave and Optical Technology Letters, vol. 43, no. 3, pp. 189-192, 2004.

[5] K. Kanjanasit and C. Wang, "A wideband resonant cavity antenna assembled using a micromachined CPW fed patch source and a two-layer metamaterial superstrate," IEEE Transactions on Components Packaging and Manufacturing Technology, vol. 9, no. 6, pp. 1142-1150, 2019.

[6] X.-Q Tian, S.-B. Liu, Y.-S. Wei, and X.-Y. Zhang, "Circularly polarized microstrip antenna with slots for beidou (compass) navigation system," in Proceedings of the 2010 International Symposium on Signals, Systems and Electronics, p. 3, Nanjing, China, September 2010.

[7] Z. Liu, S. Fang, S. Zhu et al., "BeiDou navigation terminal multi-mode asymmetric slots circularly polarized microstrip antenna," in Proceedings of 2014 3rd Asia-Pacific Conference on Antennas and Propagation (APCAP), p. 4, Harbin, China, July 2014.

[8] S.-Q. Wu, S.-B. Liu, and Z. Guo, "Coaxial probe-fed circularly polarized microstrip antenna for beidou RDSS applications," in Proceedings of the 2010 International Conference on Microwave and Millimeter Wave Technology (ICMMT), pp. 297-299, Chengdu, China, May 2010.

[9] C. Luison, A. Landini, P. Angeletti et al., "Innovative aperiodic arrays for SAR spaceborne applications," in Proceedings of the IEEE International Symposium on Phased Array Systems and Technology, pp. 959-964, Waltham, MA, USA, October 2010.

[10] A. Ossowska, J. H. Kim, and W. Wiesbeck, "Influence of mechanical antennas distortions on the performance of the HRWS SAR system," in Proceedings of the IEEE International Geoscience and Remote Sensing Symposium, pp. 2152-2155, Barcelona, Spain, July 2007.

[11] J.-F Wei, Y.-P. Song, N.-K. Fan, and B.-S. Wang, "The verification test of thermal distortion decoupling design for single panel of space micro-strip array antenna," in Proceedings of the 2006 1st International Symposium on Systems and Control in Aerospace and Astronautics, p. 4, Harbin, China, January 2006.

[12] B. Qiang, H.-J. Lee, K. L. Ford, and R. J. Langley, "Switchable textile microstrip antenna for on/off-body communications and shape distortion study," in Proceedings of the 2012 IEEE Asia-Pacific Conference on Antennas and Propagation, p. 2, Singapore, August 2012.

[13] L. Song and Y. Rahmat-Samii, "A systematic investigation of rectangular patch antenna bending effects for wearable applications," IEEE Transactions on Antennas and Propagation, vol. 66, no. 5, pp. 2219-2228, 2018.

[14] N. Adnet, I. Bruant, F. Pablo, and L. Proslier, “A mixed hexahedral finite element to model the scattering of a 
deformed microstrip antenna," Mechanics of Advanced Materials and Structures, vol. 26, no. 4, pp. 289-299, 2019.

[15] H. S. C. Wang, "Performance of phased-array antennas with mechanical errors," IEEE Transactions on Aerospace and Electronic Systems, vol. 28, no. 2, pp. 535-545, 1992.

[16] C. H. Tang, "Effects of phased array structure deformation and element outage," in Proceedings of the IEEE Antennas and Propagation Society International Symposium 1992 Digest, pp. 1608-1611, Chicago, IL, USA, June 1992.

[17] C. S. Wang, B. Y. Duan, F. S. Zhang, and M. B. Zhu, "Coupled structural-electromagnetic-thermal modelling and analysis of active phased array antennas," IET Microwaves, Antennas \& Propagation, vol. 4, no. 2, pp. 247-257, 2010.

[18] T. Takahashi, N. Nakamoto, M. Ohtsuka et al., "On-board calibration methods for mechanical distortions of satellite phased array antennas," IEEE Transactions on Antennas and Propagation, vol. 60, no. 3, pp. 1362-1372, 2012.

[19] L. R. D'Addario, "Combining loss of a transmitting array due to phase errors," The Interplanetary Network Progress Report, vol. 42-175, pp. 1-7, 2008.

[20] H. Schippers, G. Spalluto, and G. Vos, "Radiation analysis of conformal phased array antennas on distorted structures," in Proceedings of the 12th International Conference on Antennas and Propagation (ICAP 2003), pp. 160-163, Exeter, UK, April 2003.

[21] H. Rogier, F. Boeykens, and L. Vallozzi, "An efficient technique based on polynomial chaos to model the uncertainty in resonant frequency of textile antennas due to bending," IEEE Transactions on Antennas and Propagation, vol. 62, no. 3, pp. 1253-1260, 2014.

[22] L. Vallozzi, F. Boeykens, and H. Rogier, "Cylindrically-bent rectangular patch antennas: novel modeling techniques for resonant frequency variation and uncertainty," in Proceedings of the 2015 9th European Conference on Antennas and Propagation (EuCAP), p. 5, Lisbon, Portugal, April 2015.

[23] B. Ma, J. Pan, E. Wang, and Y. Luo, "Conformal bent dielectric resonator antennas with curving ground plane," IEEE Transactions on Antennas and Propagation, vol. 67, no. 3, pp. 1931-1936, 2019.

[24] S. Wang and M. S. Tong, "Mechanical deformation detection of building structures using microstrip patch antennas as sensors," IEEE Sensors Journal, vol. 18, no. 21, pp. 8676-8684, 2018.

[25] R. Elizabeth, "Microstrip antenna on kapton substrate for strain sensing applications," in Proceedings of the 16th International Conference on Advanced Communication Technology, p. 3, Pyeongchang, South Korea, February 2014.

[26] H. Huang, "Flexible wireless antenna sensor: a review," IEEE Sensors Journal, vol. 13, no. 10, pp. 3865-3872, 2013.

[27] U. Tata, H. Huang, R. L. Carter, and J. C. Chiao, "Exploiting a patch antenna for strain measurements," Measurement Science and Technology, vol. 20, no. 1, p. 7, 2009. 